\title{
Blood-based microRNAs as diagnostic biomarkers to discriminate localized prostate cancer from benign prostatic hyperplasia and allow cancer-risk stratification
}

\author{
GHADA AL-KAFAJI ${ }^{1}$, HARUN MUAYAD SAID ${ }^{2}$, MAHMOOD ABDULJALIL ALAM $^{1}$ and ZIAD TARRAQ AL NAIEB $^{3}$ \\ ${ }^{1}$ Department of Molecular Medicine and Al-Jawhara Centre for Molecular Medicine, Genetics and Inherited Disorders, \\ College of Medicine and Medical Sciences, Arabian Gulf University, Manama 329, Kingdom of Bahrain; \\ ${ }^{2}$ Department of Molecular Medicine, Graduate School of Health Sciences, Dokuz Eylul University, Izmir 35220, Turkey; \\ ${ }^{3}$ Department of Surgery, College of Medicine and Medical Sciences, Arabian Gulf University, \\ Manama 329, Kingdom of Bahrain
}

Received December 26, 2017; Accepted May 14, 2018

DOI: $10.3892 / \mathrm{ol} .2018 .8778$

\begin{abstract}
Prostate cancer (PCa) is the second most diagnosed malignancy, and the leading cause of cancer-associated mortality among males. Prostate-specific antigen (PSA) has long been used for the detection of PCa. However, PSA levels increase in $\mathrm{PCa}$ and benign prostatic hyperplasia $(\mathrm{BPH})$, and are associated with a poor disease outcome. Circulating microRNAs (miRNAs) have been determined to be highly stable in the circulation, and could be utilized as biomarkers to improve disease diagnosis and management. In the present study, the effectiveness of four $\mathrm{PCa}$-associated miRNAs in the discrimination of $\mathrm{PCa}$ from $\mathrm{BPH}$ and the risk-stratification of $\mathrm{PCa}$ was assessed. The study included 100 participants: 35 patients with localized $\mathrm{PCa}, 35$ patients with BPH and 30 healthy subjects. Patients with PCa were categorized based on their tumor stage (T), PSA level and Gleason score (GS) into low-(T 1/2, PSA $<10 \mathrm{ng} / \mathrm{ml}$ or $\mathrm{GS} \leq 7$ ) and high-risk groups (T 3/4, PSA > $20 \mathrm{ng} / \mathrm{ml}$ or $\mathrm{GS} \geq 8$ ). Reverse transcription-quantitative polymerase chain reaction was employed to assess the miRNA expression in peripheral blood samples. Significantly reduced expression of miR-15a, miR-126, miR-192 and miR-377 was observed in patients with PCa compared with patients with BPH and healthy subjects.
\end{abstract}

Correspondence to: Dr Ghada Al-Kafaji, Department of Molecular Medicine and Al-Jawhara Centre For Molecular Medicine, Genetics and Inherited Disorders, College of Medicine and Medical Sciences, Arabian Gulf University, Salmaniya Avenue, Building 293, Road 2904, Block 329, Manama 329, Kingdom of Bahrain E-mail: ghadaa@agu.edu.bh

Abbreviations: RT-qPCR, reverse transcription-quantitative polymerase chain reaction; ROC, receiver operating characteristic; AUC, area under the curve

Key words: localized prostate cancer, low-risk, high-risk, benign prostatic hyperplasia, circulating microRNA, blood biomarkers
In addition, the expression of the four miRNAs was lower in high-risk PCa patients than in low-risk PCa patients, with miR-126 being the most downregulated. The expression of the four miRNAs was also significantly and independently associated with PCa. Receiver operating characteristic curve analysis revealed a significant ability of the miRNAs to distinguish patients with $\mathrm{PCa}$ from those with $\mathrm{BPH}$, patients with PCa from controls and low-risk PCa from high-risk PCa. These data suggested that expression of these miRNAs in the blood circulation may be promising, non-invasive biomarkers for the early detection of localized PCa, and for PCa risk stratification. Further validations of the clinical implementation of these results are warranted in a larger cohort.

\section{Introduction}

Prostate cancer $(\mathrm{PCa})$ is the most common malignancy in males worldwide, and the second-leading cause of cancer-associated mortality $(1,2)$. While the prevalence of PCa in Arab countries is lower than that in Western countries, the incidence is steadily increasing (3). Although the majority of PCa cases are indolent and localized at diagnosis, localized tumors can develop into aggressive tumors in the long term (4), the early detection of PCa, when treatment is most effective, can significantly contribute to a reduction in the mortality rate. Prostate-specific antigen (PSA) has long been used for the detection and screening of PCa. However, elevated levels of PSA have also been reported in non-malignant conditions of the prostate (5), including benign prostatic hyperplasia (BPH), which is commonly misdiagnosed as $\mathrm{PCa}$, leading to unnecessary biopsies (6). PSA also has a positive predictive value of only $\sim 35 \%$, potentially leading to false-positive or false-negative results (7). It has been demonstrated that a significant proportion of males who underwent a prostate biopsy due to an elevated PSA level did not actually have PCa (8). PSA testing and screening have been demonstrated to be associated with a high rate of over-diagnosis and overtreatment in a number of clinical trials (9-11). In addition, PSA testing and 
other clinical parameters currently used for the stratification of patients with PCa, including clinical stage and Gleason score (GS) tumor grade have limitations in detecting and predicting the disease outcome (12). Therefore, PSA is neither an effective predictor of PCa, nor does it contribute to risk stratification $(13,14)$. Novel, non-invasive biomarkers that can distinguish malignant from benign prostatic tumors, and detect $\mathrm{PCa}$ at an early stage to improve disease diagnosis and management, are urgently required (15).

Genetic and epigenetic modifications contribute significantly to the pathogenicity and development of PCa (16). Among the alterations that affect the regulation of gene expression, the disturbance of microRNA (miRNA/miR) expression and function can affect several pathways involved in the initiation and development of cancer, including those of the cell cycle, proliferation, angiogenesis and apoptosis (17). The differential expression profiles of miRNAs in different types of cancer, compared with those in normal tissue, support the hypothesis that miRNAs serve a critical role in cancer (18). The upregulation and downregulation of miRNAs in different types of cancer suggest a dual role for miRNAs, acting as either tumor suppressors or oncogenes (18).

Among several miRNAs implicated in cancer, the expression levels of miR-15a, miR-126, miR-192 and miR-377 have been identified as downregulated in a wide range of cancer types, including myeloma, non-small cell lung carcinoma, pancreatic cancer, renal cell carcinoma and osteosarcoma (19-24). These four miRNAs have been described as tumor suppressors in PCa. Bonci et al (25) reported that miR-15a could function as a tumor suppressor in $\mathrm{PCa}$ via repression of cyclin D1 and Wnt family member 3A (WNT3A) oncogene expression. Song et al (26) observed that miR-126 could inhibit cancer proliferation and metastasis by targeting phosphoinositide-3-kinase regulatory subunit 2 (PIK3R2) in PCa. Sun et al (27) demonstrated that miR-192 could serve a tumor suppressive role and impair cell tumorigenicity in PCa by targeting and repressing the oncogene, nin one binding protein 1 (NOB1). Formosa et al (28) also reported that miR-377 could affect the behavior of malignant cells in PCa cell lines by targeting FZD4, a gene important in epithelial-to-mesenchymal transition.

Previous studies have proposed miRNAs as diagnostic and prognostic biomarkers for cancer $(29,30)$ and numerous other types of disease $(31,32)$, due to their high stability in bio-fluids, including blood $(33,34)$. Circulating miRNAs are considered to be either contained in exosomes, microparticles and apoptotic bodies, or associated with argonaute 2 and other RNA-binding-proteins and high-density lipoproteins, enabling their transfer from one cell to another in diverse biological processes (35). Circulating miRNA profiling studies have demonstrated differentially expressed miRNAs with diagnostic, prognostic and predictive abilities in $\mathrm{PCa}(36,37)$. The majority of these studies have evaluated the biomarker potential of these miRNAs to distinguish early stage PCa from metastatic PCa $(36,37)$. However, the present study addresses the feasibility of using circulating miRNAs as biomarkers to distinguish localized PCa from benign prostatic hyperplasia $(\mathrm{BPH})$, and to discriminate between low- and high-risk patients at an early stage.
Therefore, in the present study, the expression levels of peripheral blood miR-15a, miR-126, miR-192 and miR-377, selected based on their critical functions in $\mathrm{PCa}$, were quantified and their diagnostic value was investigated. These miRNAs may be suitable biomarkers for the early detection of localized $\mathrm{PCa}$, and for PCa risk stratification.

\section{Materials and methods}

Participants. Ethical approval to conduct the present study was obtained from the Medical Research and Ethics Committee of the College of Medicine and Medical Sciences, Arabian Gulf University (Manama, Kingdom of Bahrain). A total of 100 Bahraini participants were recruited from the Urological Clinic of King Abdullah Medical City Hospital between June 2013 and January 2014, and divided into three groups: 35 patients with localized $\mathrm{PCa}, 35$ patients with $\mathrm{BPH}$ and 30 healthy control subjects. The mean age of the patients with $\mathrm{PCa}$ was $73.8 \pm 4.2$, and the mean age of $\mathrm{BPH}$ patients was $71.8 \pm 5.9$, whereas the mean age of healthy subjects was 71.6 \pm 4.8 . All participants provided informed consent for the extraction and use of their blood samples and data. The diagnosis of localized PCa was made based on elevated PSA levels and malignant histological findings in the prostate biopsy specimen without evidence of metastatic disease. Patients with PCa were categorized into two groups, according to the D'Amico risk classification criteria for localized PCa (38), tumor (T) stage, PSA level and GS: Patients with low-risk localized PCa $(n=20)$ had a tumor stage of T1c or T2a, a PSA of $\leq 10 \mathrm{ng} / \mathrm{ml}$ or a GS of $\leq 7$; patients with high-risk localized PCa $(\mathrm{n}=15)$ had a tumor stage of T2c, a PSA of $>20 \mathrm{ng} / \mathrm{ml}$ or a GS of $\geq 8$. The diagnosis of BPH was made based on digital rectal examination, and a benign histological finding of the prostate biopsy specimen if PSA was elevated. The healthy control subjects were those who underwent routine physical examinations with no underlying prostate disease.

Blood sampling. Whole blood samples $(5 \mathrm{ml})$ were obtained from patients with $\mathrm{PCa}$ one day prior to radical prostatectomy surgery, as well as from patients with BPH and healthy control subjects. All blood samples were collected in EDTA tubes. RNAlater (1.3 ml; Ambion; Thermo Fisher Scientific, Inc., Waltham, MA, USA), an RNA stabilization reagent, was added to each tube, and the blood samples were stored at $-80^{\circ} \mathrm{C}$ until RNA extraction.

Selection of miRNAs as candidate blood biomarkers. According to previous reports, a number of PCa-associated miRNAs were selected, including miR-15a, miR-126, miR-192 and miR-377, to evaluate their potential as diagnostic biomarkers for PCa. The 4 selected miRNAs were previously reported to be dysregulated in prostate tumor tissue and $\mathrm{PCa}$ cell lines (25-28).

miRNA extraction and reverse transcription $(R T)$. The extraction of RNA, including small RNA, was performed using the miRNeasy kit (Qiagen GmbH, Hilden, Germany) according to the manufacturer's protocols, as previously described $(30,31,39)$. The quality and concentration of RNA extracted from the clinical samples was evaluated using 
Table I. Clinical characteristics of participants.

\begin{tabular}{|c|c|c|c|c|c|}
\hline Characteristic & All PCa & Low-risk PCa & High-risk PCa & $\mathrm{BPH}$ & Healthy \\
\hline Total number & 35 & 20 & 15 & 35 & 30 \\
\hline Age, years & $73.8 \pm 4.2$ & $73.0 \pm 4.6$ & $74.9 \pm 3.5$ & $71.8 \pm 5.9$ & $71.6 \pm 4.8$ \\
\hline PSA, ng/ml & $15.6 \pm 7.6^{\mathrm{a}}$ & $9.15 \pm 0.51$ & $21.7 \pm 5.98^{b}$ & $8.7 \pm 1.5$ & $3.7 \pm 0.7$ \\
\hline \multicolumn{6}{|l|}{ Gleason score } \\
\hline$\leq 7$ & $20(57.1)$ & $20(100)$ & & - & - \\
\hline$\geq 8$ & $15(42.9)$ & & $15(100)$ & - & - \\
\hline \multicolumn{6}{|l|}{ Tumor stage } \\
\hline $\mathrm{T} 1 / 2$ & $20(57.1)$ & $20(100)$ & & - & - \\
\hline $\mathrm{T} 3 / 4$ & $15(42.9)$ & & $15(100)$ & - & - \\
\hline \multicolumn{6}{|c|}{ Lymph node stage } \\
\hline No & $32(91.4)$ & $20(100)$ & & - & - \\
\hline N1 & $3(8.6)$ & & $3(20)$ & - & - \\
\hline \multicolumn{6}{|c|}{ Metastasis stage } \\
\hline M0 & $35(100)$ & $20(100)$ & $15(100)$ & - & - \\
\hline M1 & - & - & - & - & - \\
\hline
\end{tabular}

${ }^{\mathrm{a}} \mathrm{P}<0.05$ vs. $\mathrm{BPH}$ and controls; ${ }^{\mathrm{b}} \mathrm{P}<0.05$ vs. low-risk PCa. Data are presented as $\mathrm{n}$, the mean \pm standard deviation or $\mathrm{n}(\%)$ as appropriate. $\mathrm{PCa}$, prostate cancer; $\mathrm{BPH}$, benign prostatic hyperplasia; PSA, prostate-specific antigen.

a Nanodrop ND-100 spectrophotometer (Thermo Fisher Scientific, Inc.). The concentrations of RNA ranged between 50 and $75 \mathrm{ng} / \mu \mathrm{l}$; aliquots from all RNA samples were diluted to identical final concentrations of $20 \mathrm{ng} / \mu 1$. cDNA was prepared from $20 \mathrm{ng}$ RNA using a TaqMan miRNA Reverse Transcription kit (Applied Biosystems; Thermo Fisher Scientific, Inc.) as previously described $(30,31,39)$, with specific stem-loop RT primers. All cDNA samples were stored at $-20^{\circ} \mathrm{C}$ until further analysis.

Reverse transcription-quantitative polymerase chain reaction (RT-qPCR). The expression of miRNAs was quantified by RT-qPCR using TaqMan Universal PCR Master mix II (Applied Biosystems; Thermo Fisher Scientific, Inc.). The kit contained $1.33 \mu \mathrm{l}$ cDNA, $10 \mu \mathrm{l}$ TaqMan 2X Universal PCR Master mix II (Applied Biosystems; Thermo Fisher Scientific, Inc.), $1 \mu \mathrm{l}$ gene-specific primers and $7.67 \mu$ l nuclease-free water to a final volume of $20 \mu 1$. RT-qPCR was performed on a 7900HT Real Time PCR system (Applied Biosystems; Thermo Fisher Scientific, Inc.). The PCR primer sequences for the targets were as follows: miR-15a, 5'-UAGCAGCAC AUAAUGGUUUGUG-3'; miR-126, 5'-UCGUACCGUGAG UAAUAAUGC-3'; miR-192, 5'-CUGCCAAUUCCAUAG GUCACAG-3'; and miR-377, 5'-AUCACACAAAGGCAA CUUUUGU-3'. The PCR primer sequence for reference was as follows: U6B, 5'-CGCAAGGATGACACGCAAATTCGT GAAGCGTTCCATATTTTT-3'.

The qPCR was performed using the following cycling conditions: $95^{\circ} \mathrm{C}$ for $10 \mathrm{~min}$, followed by $95^{\circ} \mathrm{C}$ for $15 \mathrm{sec}$ and $60^{\circ} \mathrm{C}$ for $60 \mathrm{sec}$ for a total of 40 cycles. The $2^{-\Delta \Delta \mathrm{Cq}}$ method was used to determine the expression of miRNAs relative to U6B (40) using SDS software version 1.4 (Applied Biosystems; Thermo Fisher Scientific, Inc.). The mean fold change of duplicate qPCR amplifications was used in statistical analysis.
Statistical analysis. Statistical analysis was performed using SPSS version 23 (IBM Corp., Armonk, NY, USA). Differences in the clinical variables and the relative expression of blood miRNAs between patients with PCa and those with BPH, as well as between patients with low-risk and high-risk localized PCa were analyzed with Student's t test. Comparisons of the clinical variables among more than 2 groups were evaluated using one-way analysis of variance. Data for miRNA expression, age and PSA are presented as the mean \pm standard deviation. Data for GS, tumor stage, lymph node stage and metastasis stage are presented as numbers and percentages. The association between individual miRNAs and PCa was determined with multivariate logistic regression analysis by obtaining the odds ratios (OR) and $95 \%$ confidence intervals (CIs) for either a crude model (1a and 2a), an age-adjusted model (1b), or an age- and PSA-adjusted model (2b). Receiver operating characteristic (ROC) curves and the area under the ROC curve (AUC) were used to assess the diagnostic accuracy of miRNAs as biomarkers. $\mathrm{P}<0.05$ was considered to represent a statistically significant difference.

\section{Results}

Clinical characteristics of participants. Table I lists the clinical characteristics of the participants, including 35 patients with localized PCa, 35 patients with BPH and 30 healthy control subjects. Patients with localized PCa were divided into two groups: Low-risk $(n=20)$ and high-risk $(n=15)$, based on T stage, serum PSA and GS (35).

As shown in Table I, there was no significant difference in the mean age among the three subjects groups $(\mathrm{P}>0.05)$. Patients with PCa or BPH had significantly higher levels of serum PSA, with mean values of 15.6 and $8.7 \mathrm{ng} / \mathrm{ml}$, respectively, compared with the healthy subjects $(3.7 \mathrm{ng} / \mathrm{ml} ; \mathrm{P}<0.05)$. 
Furthermore, the mean serum PSA level was significantly higher in patients with $\mathrm{PCa}$ than in patients with $\mathrm{BPH}(\mathrm{P}<0.05)$.

In the localized PCa group, 20 patients presented with low-risk PCa, with a tumor stage of T1c or T2a, a PSA of $\leq 10 \mathrm{ng} / \mathrm{ml}$ and a GS of $\leq 7$ and 15 patients presented with high-risk PCa, with a tumor stage of T2c or greater, a PSA of $>20 \mathrm{ng} / \mathrm{ml}$ and a GS of $\geq 8$. There was no significant difference in mean age between the groups $(\mathrm{P}>0.05)$. The mean serum PSA levels differed significantly $(\mathrm{P}<0.05)$ and were higher in the high-risk group than in the low-risk group. Three patients in the high-risk group presented with regional lymph node involvement (stage N1), and none of the patients presented with distant metastasis.

Expression of blood miRNAs in patients with localized $P C a$, patients with BPH and healthy subjects. Using RT-qPCR, the expression of miR-15a, miR-126, miR-192 and miR-377 relative to the internal control U6B were quantitated in peripheral whole blood and compared between patients with localized $\mathrm{PCa}$ or BPH, and healthy control subjects.

Fig. 1 demonstrates the overall expression of the 4 miRNAs, which were all significantly lower in patients with $\mathrm{PCa}$ than in patients with $\mathrm{BPH}$ or healthy subjects $(\mathrm{P}<0.05)$. miR-15a expression was lower in patients with $\mathrm{PCa}(0.54 \pm 0.1)$ compared with either patients with BPH $(2.44 \pm 0.47)$ or healthy subjects (2.35 \pm 0.4$)$. miR-126 expression was also lower in patients with PCa $(0.97 \pm 0.88)$ with respect to patients with BPH $(4.53 \pm 1.0)$ and healthy subjects $(5.85 \pm 1.5)$. Similarly, patients with PCa demonstrated a decreased expression of miR-192 (1.61 \pm 0.45$)$ compared with patients with BPH $(6.85 \pm 1.4)$ or healthy subjects (7.93 \pm 1.6$)$. Finally, patients with PCa exhibited a lower expression of miR-377 $(0.64 \pm 0.07)$ compared with patients with $\mathrm{BPH}$ $(3.04 \pm 0.5)$ or healthy subjects $(3.34 \pm 0.7)$.

Expression of blood miRNAs in low-risk and high-risk patients with localized PCa. A subgroup analysis was performed to determine whether the expression of miR-15a, miR-126, miR-192 and miR-377 differed between patients with localized PCa categorized as low-risk (T1c or T2a, PSA $\leq 10 \mathrm{ng} / \mathrm{ml}$ and GS $\leq 7$ ) or high-risk (T2c or greater, PSA $>20 \mathrm{ng} / \mathrm{ml}$ and GS $\geq 8$ ) using the D'Amico classification. As illustrated in Fig. 2, expression levels of the 4 miRNAs were significantly $(\mathrm{P}<0.05)$ lower in patients with high-risk localized $\mathrm{PCa}$ than in patients with low-risk localized PCa.

miR-15a expression was 6.3 -fold lower in high-risk PCa $(0.11 \pm 0.24)$ than in low-risk PCa $(0.88 \pm 0.34)$. miR-126 expression was 15.5 -fold lower in high-risk PCa $(0.11 \pm 0.17)$ than in low-risk PCa (1.7 \pm 0.45$)$. miR-192 expression was 5.6-fold lower in high-risk PCa $(0.46 \pm 0.17)$ than in low-risk PCa (2.58 $\pm 0.5 ; \mathrm{P}=0.01)$. miR-377 expression was lower by 2.2 -fold in high-risk PCa $(0.39 \pm 0.3)$ than in low-risk PCa $(0.86 \pm 0.4)$. Amongst the 4 miRNAs analyzed, miR-126 was the most downregulated.

Multivariate regression analysis of association. Associations between the expression of blood miR-15a, miR-126, miR-192 and miR-377 and the presence of localized PCa were further analyzed. Analysis using multivariate logistic regression models based on single miRNAs confirmed a significant, independent association of each miRNA with PCa. When the

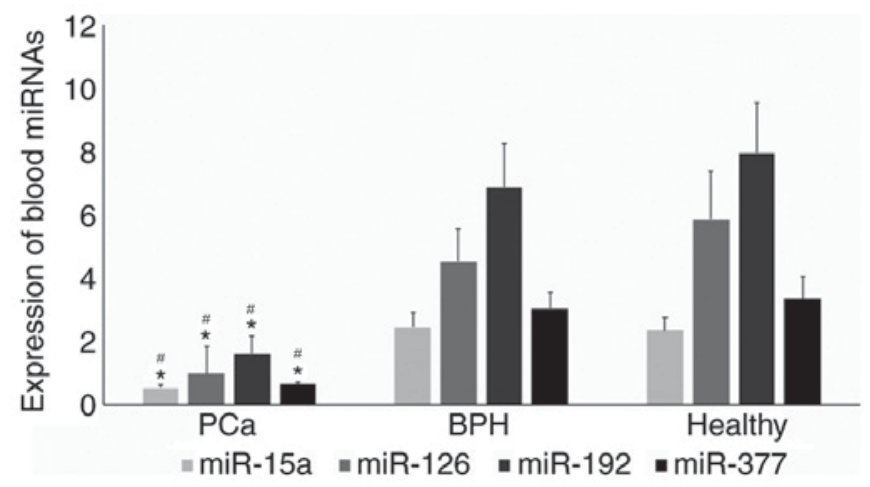

Figure 1. Expression of blood miRNAs in patients with localized PCa, patients with BPH and healthy subjects. Expression of blood miR-15a, miR-126, miR-192 and miR-377 relative to the internal control, U6B, were quantitated by reverse transcription-quantitative polymerase chain reaction in peripheral whole blood and compared in patients with localized PCa $(n=35)$, patients with BPH $(n=35)$ and healthy control subjects $(n=30)$. The relative quantification value for each miRNA was expressed as the mean \pm standard deviation. ${ }^{*} \mathrm{P}<0.05$ vs. $\mathrm{BPH}$ and ${ }^{\#} \mathrm{P}<0.05$ vs. healthy. miRNA/miR, microRNA; PCa, prostate cancer; $\mathrm{BPH}$, benign prostatic hyperplasia.

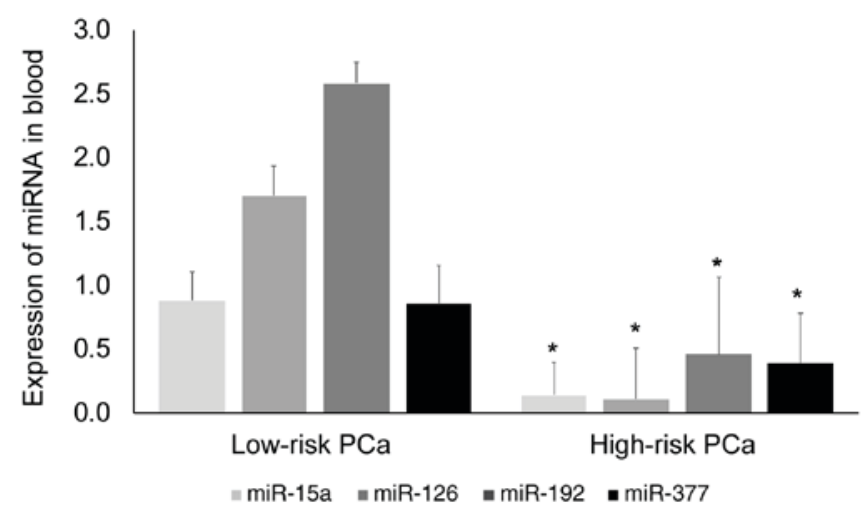

Figure 2. Expression of blood miRNAs in low-risk and high-risk patients with localized PCa. Expression of blood miR-15a, miR-126, miR-192 and miR-377 quantified by reverse transcription-quantitative polymerase chain reaction were sub-analyzed in patients with localized PCa categorized as low-risk or high-risk of tumor aggressiveness using D'Amico scores. The relative quantification value for each miRNA was expressed as the mean \pm standard deviation. ${ }^{*} \mathrm{P}<0.05$ vs. low-risk localized $\mathrm{PCa}$. miRNA/miR, microRNA; PCa, prostate cancer.

group of healthy control subjects was used as the reference category, (Table II), crude and age-adjusted regression models demonstrated that the 4 miRNAs were significantly and independently associated with $\mathrm{PCa}(\mathrm{P}<0.05)$, whereas none of the miRNAs were significantly associated with $\mathrm{BPH}(\mathrm{P}>0.05)$. In addition, when the group of patients with BPH was used as the reference category, the association of the four miRNAs with the presence of $\mathrm{PCa}$ was statistically significant $(\mathrm{P}<0.05)$ prior to and following adjustment for age and PSA (Table III).

Discriminative ability of miRNAs for localized PCa. To determine the discriminative ability of blood miR-15a, miR-126, miR-192 and miR-377 for localized PCa, ROC curve analysis was performed and the AUC was reported for each miRNA.

The ROC analysis was first applied for the four miRNAs in patients with PCa vs. patients with BPH. The results 
Table II. Multivariate logistic regression analysis of miRNAs for discriminating PCa in the healthy control group reference category (Model 1).

\begin{tabular}{|c|c|c|c|c|c|c|}
\hline \multirow[b]{2}{*}{ miRNA } & \multicolumn{3}{|c|}{$\mathrm{PCa}$} & \multicolumn{3}{|c|}{$\mathrm{BPH}$} \\
\hline & OR & $95 \%$ CI & P-value & OR & $95 \% \mathrm{CI}$ & P-value \\
\hline \multicolumn{7}{|l|}{$\operatorname{miR}-15 \mathrm{a}$} \\
\hline $1 \mathrm{a}$ & 0.39 & $0.21-0.72$ & 0.003 & 1.01 & $0.84-1.23$ & 0.89 \\
\hline $1 b$ & 0.34 & $0.21-0.74$ & 0.004 & 1.02 & $0.84-1.24$ & 0.86 \\
\hline \multicolumn{7}{|l|}{ miR-126 } \\
\hline $1 \mathrm{a}$ & 0.67 & $0.53-0.85$ & 0.001 & 0.97 & 0.91-1.04 & 0.44 \\
\hline $1 b$ & 0.69 & $0.55-0.87$ & 0.002 & 0.97 & $0.90-1.03$ & 0.45 \\
\hline \multicolumn{7}{|l|}{ miR-192 } \\
\hline $1 \mathrm{a}$ & 0.77 & $0.64-0.93$ & 0.005 & 0.71 & $0.95-1.01$ & 0.71 \\
\hline $1 b$ & 0.76 & 0.64-0.92 & 0.004 & 0.99 & $0.95-1.11$ & 0.69 \\
\hline \multicolumn{7}{|l|}{ miR-377 } \\
\hline $1 \mathrm{a}$ & 0.51 & $0.31-0.85$ & 0.009 & 0.98 & $0.87-1.11$ & 0.76 \\
\hline $1 b$ & 0.50 & $0.30-0.84$ & 0.009 & 0.97 & $0.83-1.20$ & 0.77 \\
\hline
\end{tabular}

1a, crude model; 1b, age-adjusted model; miRNA/miR, microRNA; PCa, prostate cancer; BPH, benign prostatic hyperplasia; OR, odds ratio; CI, confidence interval.

Table III. Multivariate logistic regression analysis of miRNAs for the presence of prostate cancer in the benign prostatic hyperplasia group reference category (Model 2).

\begin{tabular}{lccc}
\hline & \multicolumn{3}{c}{$95 \%$ confidence } \\
miRNA & Odds ratio & P-value \\
\hline miR-15a & & $0.25-0.80$ & 0.013 \\
$2 \mathrm{a}$ & 0.45 & $0.13-0.95$ & 0.024 \\
$2 \mathrm{~b}$ & 0.35 & & \\
$\mathrm{miR}-126$ & & $0.46-0.78$ & 0.001 \\
$2 \mathrm{a}$ & 0.60 & $0.35-0.83$ & 0.046 \\
$2 \mathrm{~b}$ & 0.54 & & \\
miR-192 & & $0.49-0.82$ & 0.001 \\
$2 \mathrm{a}$ & 0.63 & $0.49-0.89$ & 0.030 \\
$2 \mathrm{~b}$ & 0.66 & & \\
miR-377 & & $0.27-0.81$ & 0.008 \\
$2 \mathrm{a}$ & 0.46 & $0.13-0.97$ & 0.040 \\
$2 \mathrm{~b}$ & 0.36 & & \\
\hline
\end{tabular}

2a, crude model; 2b, age- and PSA-adjusted model; OR, odds ratio.

demonstrated good diagnostic capabilities in discriminating between the groups, with an AUC of $0.744(\mathrm{P}<0.001)$, $0.844(\mathrm{P}<0.001), 0.82(\mathrm{P}<0.001)$ and $0.73(\mathrm{P}=0.001)$ for $\mathrm{miR}-15 \mathrm{a}$, miR-126, miR-192 and miR-377, respectively (Fig. 3A and Table IV).

Likewise, when the ROC analysis was applied for the 4 miRNAs in patients with PCa vs. healthy subjects (Fig. 3B and Table IV), the results revealed significant diagnostic abilities in differentiating between the groups,
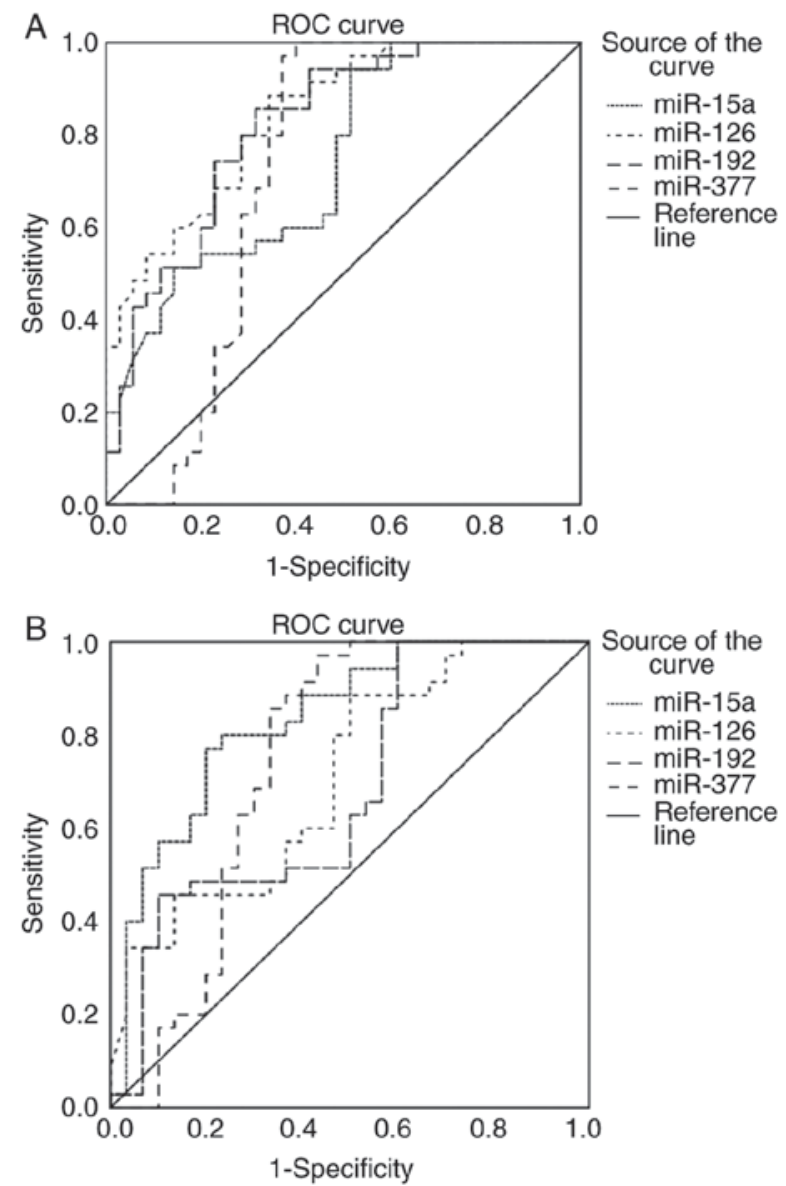

Figure 3. Discriminative ability of miRNAs for localized PCa. The abilities of blood miR-15a, miR-126, miR-192 and miR-377 as biomarkers for localized PCa were obtained using the ROC curve analysis and the area under the curve was reported for each miRNA. (A) Patients with localized PCa vs. patients with benign prostatic hyperplasia. (B) Patients with localized PCa vs. healthy subjects. miRNA/miR, microRNA; PCa, prostate cancer; ROC, receiver operating characteristic. 
Table IV. Discriminative ability of miRNAs for localized PCa.

\begin{tabular}{|c|c|c|c|c|c|c|}
\hline \multirow[b]{2}{*}{ miRNA } & \multicolumn{3}{|c|}{ PCa vs. benign prostatic hyperplasia } & \multicolumn{3}{|c|}{ PCa vs. healthy } \\
\hline & AUC & $95 \% \mathrm{CI}$ & P-value & AUC & $95 \% \mathrm{CI}$ & P-value \\
\hline $\operatorname{miR}-15 a$ & 0.744 & $0.63-0.86$ & $<0.001$ & 0.79 & $0.67-0.90$ & $<0.001$ \\
\hline $\operatorname{miR}-126$ & 0.844 & $0.76-0.93$ & $<0.001$ & 0.70 & $0.56-0.82$ & 0.009 \\
\hline miR-192 & 0.82 & $0.72-0.92$ & $<0.001$ & 0.66 & $0.52-0.78$ & 0.024 \\
\hline miR-377 & 0.73 & $0.61-0.86$ & 0.001 & 0.75 & $0.63-0.87$ & $<0.001$ \\
\hline
\end{tabular}

miRNA/miR, microRNA; PCa, prostate cancer; AUC, area under the curve; CI, confidence interval.

with an AUC of $0.79(\mathrm{P}<0.001), 0.70(\mathrm{P}=0.009)$, and 0.75 $(\mathrm{P}<0.001)$ for miR-15a, miR-126 and miR-377, respectively, and a more moderate but significant ability for miR-192 (AUC, 0.66; P=0.024).

Discriminative ability of miRNAs for PCa risk stratification. Next, the abilities of blood miR-15a, miR-126, miR-192 and miR-377 to accurately risk-stratify patients with localized PCa were evaluated. The ROC analysis was applied to patients with localized PCa categorized as low-risk or high-risk using the D'Amico classification.

As demonstrated in Fig. 4 and Table V, the 4 miRNAs displayed high diagnostic capabilities for differentiating between low- and high-risk PCa. The AUCs were 0.92 $(\mathrm{P}<0.001), 0.91(\mathrm{P}<0.001), 0.87, \mathrm{P}<0.001)$ and $0.86(\mathrm{P}<0.001)$ for miR-15a, miR-126, miR-192 and miR-377, respectively. Notably, the highest predictive values were obtained for miR-15a and miR-126.

\section{Discussion}

The introduction of PSA testing into clinical practice has aided the early detection and screening of $\mathrm{PCa}$ (2). However, despite the high sensitivity of PSA, the test is not specific for PCa $(5,7,13,14)$, as PSA levels may also increase in non-malignant conditions of the prostate, including BPH; this has led to over-diagnosis and over-treatment $(5,6)$. Furthermore, PSA levels are poorly correlated with tumor aggressiveness and poorer disease outcomes (15). The limitations in the diagnostic accuracy of PSA raise an urgent requirement for alternative rapid and accurate markers for the detection of $\mathrm{PCa}$, and the identification of high-risk patients at an early stage. The aberrant expression of miRNAs in various physiopathological conditions has led to the intense investigation of their potential role as a novel class of markers (17). As they are highly stable in human biofluids $(33,34)$, miRNAs exhibit promise as non-invasive biomarkers for a variety of diseases $(31,32,39)$. Circulating miRNAs secreted by tumor cells into the blood may reflect the tumor of origin (41) and the extent of tumor progression, including in $\mathrm{PCa}(42,43)$, thus representing promising diagnostic or prognostic disease biomarkers (29-31,36).

Among the growing number of miRNAs that have been identified as serving a key role in cancer, the expression levels of miR-15a, miR-126, miR-192 and miR-377 have been frequently identified as downregulated in a wide
Table V. Discriminative ability of miRNAs for localized PCa-risk stratification.

\begin{tabular}{lccc}
\hline & \multicolumn{3}{c}{ Low-risk vs. high-risk PCa } \\
\cline { 2 - 4 } miRNA & $\begin{array}{c}\text { Area under } \\
\text { the curve }\end{array}$ & $\begin{array}{c}\text { 95\% confidence } \\
\text { interval }\end{array}$ & P-value \\
\hline miR-15a & 0.92 & $0.83-1.00$ & $<0.001$ \\
miR-126 & 0.91 & $0.8-1.00$ & $<0.001$ \\
miR-192 & 0.87 & $0.75-0.99$ & $<0.001$ \\
miR-377 & 0.86 & $0.73-0.98$ & $<0.001$ \\
\hline
\end{tabular}

$\mathrm{PCa}$, prostate cancer; miRNA/miR, microRNA.

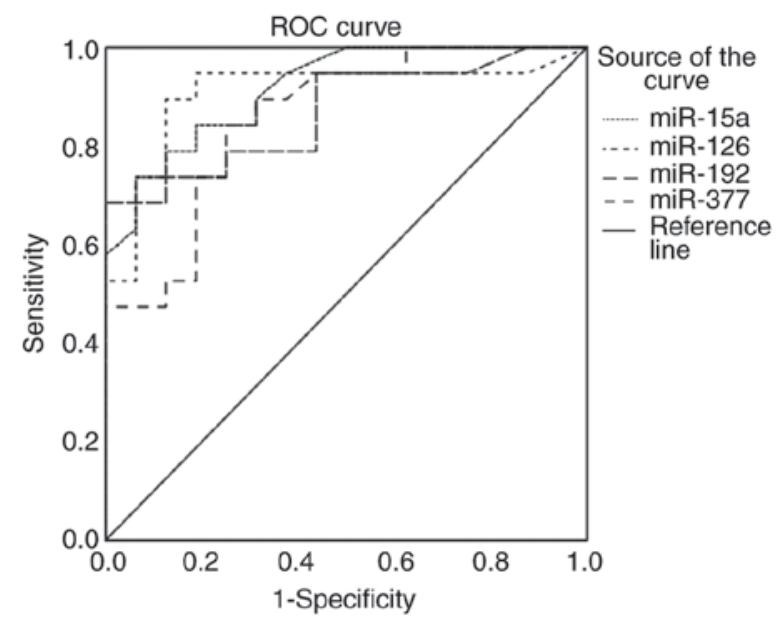

Figure 4. Discriminative ability of miRNAs for PCa risk stratification. The abilities of blood miR-15a, miR-126, miR-192 and miR-377 to accurately risk-stratify patients with localized PCa were determined using the ROC curve analysis applied in patients with localized PCa categorized as low-risk or high-risk of tumor aggressiveness using D'Amico scores. miRNA/miR, microRNA; PCa, prostate cancer; ROC, receiver operating characteristic.

range of cancer types (19-24). Particularly in PCa, these miRNAs have been described as tumor suppressors that target multiple oncogenes (25-28). Since the majority of studies have focused on the potential value of miRNAs as biomarkers for advanced and metastatic PCa $(36,37)$, the present study focused on the utility of circulating miRNAs as biomarkers to distinguish localized PCa from BPH, and 
also to discriminate between patients with low- and high-risk localized PCa. Therefore, the expression levels of peripheral blood miR-15a, miR-126, miR-192 and miR-377, selected based on their critical functions in PCa (25-28), were quantified, and their diagnostic value investigated; they may be novel biomarkers for the early detection of localized PCa, and for PCa risk stratification.

Significantly lower expression of miR-15a, miR-126, miR-192 and miR-377 was identified in patients with PCa, compared with in patients with BPH or healthy subjects (Fig. 1). Subgroup analysis of patients with localized PCa categorized as high- or low-risk using the D'Amico classification (38) revealed that the four miRNAs investigated were significantly lower in patients with high-risk PCa than in patients with low-risk PCa (Fig. 2). Among the miRNAs, miR-126 was the most downregulated, with a 15.5 -fold decrease in patients with high-risk PCa vs. in patients with low-risk PCa (Fig. 2). These results may suggest that miR-15a, miR-126, miR-192 and miR-377 participate in the pathogenicity and development of PCa. Furthermore, analysis using multivariate logistic regression models based on single miRNAs confirmed a significant, independent association between each of the 4 miRNAs and $\mathrm{PCa}$, with or without adjustments for age, whereas none of the miRNAs were significantly associated with BPH (Table II). When these models were applied in multivariate logistic regression analysis using patients with $\mathrm{BPH}$ as the reference category, the 4 miRNAs were significantly associated with PCa even after adjustment for age and PSA (Table III). These data indicated that the reduced expression of circulating miR-15a, miR-126, miR-192 and miR-377 in the blood may represent predictive biomarkers for the presence of $\mathrm{PCa}$. To the best of our knowledge, this is the first study to identify the downregulation of blood miR-15a, miR-126, miR-192 and miR-377 in patients with localized PCa, compared with in patients with BPH and healthy individuals, and to demonstrate that these four miRNAs are predictive for PCa.

miR-15a is located near miR-16-1 at chromosome $13 q 14$, a region that is frequently altered in cancer (19). miR-15a serves an important role in cell differentiation, proliferation, apoptosis and angiogenesis (19). In PCa, miR-15a was reported to target and suppress the expression of the cyclin D1 and WNT3A oncogenes (25).

miR-126 (also referred to as miR-126-3p) and its complement miR-126* (miR-126-5p) are derived from the epidermal growth factor-like domain 7 gene on chromosome 9 located within intron $7(44,45)$. It is highly expressed in vascular endothelial cells, and represses negative regulators of vascular endothelial growth factor signaling pathways, thus promoting pro-angiogenic processes and enhancing blood vessel formation $(44,45)$. The function of miR-126 as a tumor suppressor was proposed based on its ability to inhibit cancer cell growth, adhesion, migration and invasion (20,46-49). Song et al (26) identified that miR-126 could inhibit cancer proliferation and metastasis by targeting PIK3R2 in PCa. However, miR-126 may also act as an oncogene by promoting blood vessel formation and supporting cancer progression (50). These observations suggested that the behavior of miR-126 in cancer biology is complex, indicating that the role of miR-126 in different tissue types may vary, as miR-126 mediates different signaling pathways in different tissues (51).
miR-192, another cancer regulator, was demonstrated to impair cell tumorigenicity in PCa by targeting and repressing the oncogene NOB1 (27).

miR-377 could also affect the malignant cell behavior of PCa cell lines by targeting FZD4, an important gene in the epithelial-to-mesenchymal transition (28). The downregulation of miR-377 was also reported to be associated with a higher PSA and Gleason score, and lymph node invasion, in specimens of primary and metastatic prostate tumors (28).

In the present study, the downregulation of blood miR-15a, miR-126, miR-192 and miR-377 in patients with localized PCa compared with in patients with BPH and healthy controls supports the hypothesis that these miRNAs may function as tumor suppressers and have an important role in disease pathogenicity. Additionally, the low blood expression of these four miRNAs in patients with high-risk localized PCa compared patients with low-risk localized PCa may suggest that they serve a role in tumor aggressiveness.

The results of the present study indicated that blood miR-15a, miR-126, miR-192 and miR-377 levels could be used to discriminate PCa from BPH with high diagnostic accuracy, as determined by ROC analysis (Fig. 3A and Table IV). In the differentiation between patients with $\mathrm{PCa}$ and healthy subjects, miR-15a, miR-126 and miR-377 displayed relatively high diagnostic values, while miR-192 exhibited a more moderate, though significant, ability (Fig. 3B and Table IV). Of note, ROC analysis demonstrated the significant diagnostic power of miR-15a, miR-126, miR-192 and miR-377 to discriminate patients with low-risk localized $\mathrm{PCa}$ from patients with high-risk localized $\mathrm{PCa}$, and miR-15a and miR-126 were the best predictors of high-risk disease (Fig. 4 and Table V). A previous study by Watahiki et al (37) demonstrated the decreased expression of miR-126, and other miRNAs, including miR151-3p, miR423-3p, miR152 and miR-21, in the plasma of patients with localized PCa. However, in their study, only the combination of these miRNAs differentiated the two groups of patients rather than any individual miRNA (37).

Peripheral blood is easily accessible and its extraction is non-invasive. The development of miRNA biomarkers that allow the differentiation between patients with malignant and benign prostate tumors could aid in avoiding invasive biopsies in Patients with BPH (15). Additionally, miRNA biomarkers for indolent localized $\mathrm{PCa}$, which may remain unrecognized for a number of years, could allow early diagnosis when the cancer is treatable and had not progressed to a more clinically significant disease (15).

The present study clearly demonstrated that expression of miR-15a, miR-126, miR-192 and miR-377 could be developed as blood-based non-invasive biomarkers to distinguish PCa from BPH, and to identify patients with low-risk and high-risk localized PCa. The small sample size in the present study may limit the statistical power of the results, and further validation studies for the clinical implementation of the results are warranted with a larger sample size. Furthermore, the present study focused solely on the potential of miR-15a, miR-126, miR-192 and miR-377 as biomarkers for the early detection of localized PCa or PCa risk stratification. Additional studies are underway in our laboratory to 
investigate the clinical significance of other PCa-associated miRNA biomarkers.

Taken together, the results of the present study revealed that the expression of circulating miR-15a, miR-126, miR-192 and miR-377 in blood was significantly lower in patients with localized PCa than in patients with BPH and healthy subjects, lower in patients with high-risk localized PCa than in patients with low-risk localized PCa, and independently associated with the occurrence of localized PCa. These results demonstrated that the expression of miR-15a, miR-126, miR-192 and miR-377 holds promise as early blood-based biomarkers for determining the presence of localized $\mathrm{PCa}$, and for $\mathrm{PCa}$ risk assessment.

\section{Acknowledgements}

Not applicable.

\section{Funding}

The present study was supported by a research grant from the College of Medicine and Medical Sciences, Arabian Gulf University (grant no. 93).

\section{Availability of data and materials}

The analyzed data sets generated during the study are available from the corresponding author, on reasonable request.

\section{Authors' contributions}

GAK was responsible for project development, data management, data analysis, manuscript writing and manuscript editing. HMS was responsible for project development, data management and manuscript writing. MAA was responsible for data collection and data analysis. ZTAN was responsible for data collection and manuscript writing. All authors have read and approved the manuscript.

\section{Ethical approval and consent to participate}

Ethical approval to conduct the present study was obtained from the Medical Research and Ethics Committee of the College of Medicine and Medical Sciences, Arabian Gulf University. All participants provided written informed consent for the use of their blood samples and data.

\section{Consent for publication}

Not applicable.

\section{Competing interests}

The authors declare that they have no competing interests.

\section{References}

1. Greenlee RT, Hill-Harmon MB, Murray T and Thun M: Cancer statistics, 2001. CA Cancer J Clin 51: 15-36, 2001.

2. Jemal A, Bray F, Center MM, Ferlay J, Ward E and Forman D: Global cancer statistics. CA Cancer J Clin 61: 69-90, 2011.
3. Hilal L, Shahit M, Mukherji D, Charafeddine M, Farhat Z, Temaraz S, Khauli R and Shamseddine A: Prostate cancer in the Arab world: A view from the inside. Clin Genitourin Cancer 13: 505-511, 2015.

4. Popiolek M, Rider JR, Andrén O, Andersson SO, Holmberg L, Adami HO and Johansson JE: Natural history of early, localized prostate cancer: A final report from three decades of follow-up. Eur Urol 63: 428-435, 2013.

5. Hoffman RM, Gilliland FD, Adams-Cameron M, Hunt WC and Key CR: Prostate-specific antigen testing accuracy in community practice. BMC Fam Pract 3: 19, 2002.

6. Nadler RB, Humphrey PA, Smith DS, Catalona WJ and Ratliff TL: Effect of inflammation and benign prostatic hyperplasia on elevated serum prostate specific antigen levels. J Urol 154: 407-413, 1995.

7. Adhyam M and Gupta AK: A review on the clinical utility of PSA in cancer prostate. Indian J Surg Oncol 3: 120-129, 2012.

8. Boddy JL, Pike DJ and Malone PR: A seven-year follow up of men following a benign prostate biopsy. Eur Urol 44: 17-20, 2003.

9. Hugosson J, Carlsson S, Aus G, Bergdahl S, Khatami A, Lodding P, Pihl CG, Stranne J, Holmberg E and Liljia H: Mortality results from the Göteborg randomised population-based prostate-cancer screening trial. Lancet Oncol 11: 725-732, 2010.

10. Schröder FH, Hugosson J, Roobol MJ, Tammela TL, Ciatto S, Nelen V, Kwiatkowski M, Lujan M, Lilja H, Zappa M, et al: Screening and prostate-cancer mortality in a randomized european study. N Engl J Med 360: 1320-1328, 2009.

11. Andriole GL, Crawford ED, Grubb RL III, Buys SS, Chia D, Church TR, Fouad MN, Gelmann EP, Kuale PA, Reding DJ, et al: Mortality results from a randomized prostate-cancer screening trial. N Engl J Med 360: 1310-1319, 2009.

12. Stamey TA, McNeal JE, Yemoto CM, Sigal BM and Johnstone IM: Biological determinants of cancer progression in men with prostate cancer. JAMA 281: 1395-1400, 1999.

13. Oesterling JE: Prostate specific antigen: A critical assessment of the most useful tumor marker for adenocarcinoma of the prostate. J Urol 145: 907-923, 1991.

14. Thompson IM, Pauler DK, Goodman PJ, Tangen CM, Lucia MS, Parnes HL, Minasian LM, Ford LG, Lippman SM, Crawford ED, et al: Prevalence of prostate cancer among men with a prostate-specific antigen level $<0 r=4.0 \mathrm{ng}$ per milliliter. $\mathrm{N}$ Engl J Med 350: 2239-2246, 2004.

15. Velonas VM, Woo HH, Remedios CG and Assinder SJ: Current status of biomarkers for prostate cancer. Int J Mol Sci 14: 11034-11060, 2013.

16. Kgatle MM, Kalla AA, Islam MM, Sathekge M and Moorad R: Prostate cancer: Epigenetic alterations, risk factors, and therapy. Prostate Cancer 2016: 5653862, 2016.

17. Kloosterman WP and Plasterk RH: The diverse functions of microRNAs in animal development and disease. Dev Cell 11: 441-450, 2006.

18. Zhang B, Pan X, Cobb GP and Anderson TA: microRNAs as oncogenes and tumor suppressors. Dev Biol 302: 1-12, 2007.

19. Li F, Xu Y, Deng S, Li Z, Zou D, Yi S, Sui W, Hao M and Qiu L: MicroRNA-15a/16-1 cluster located at chromosome $13 \mathrm{q} 14$ is down-regulated but displays different expression pattern and prognostic significance in multiple myeloma. Oncotarget 6: 38270-38282, 2015.

20. Ebrahimi F, Gopalan V, Smith RA and Lam AK: miR-126 in human cancers: Clinical roles and current perspectives. Exp Mol Pathol 96: 98-107, 2014.

21. Yunxia Z and Hongying D: Low expression of miR-192 in NSCLC and its tumor suppressor functions in metastasis via targeting ZEB2. Open Life Sci 11: 293-297, 2016.

22. Botla SK, Savant S, JandaghiP, Bauer AS, Mücke O, MoskalevEA, Neoptolemos JP, Costello E, Greenhalf W, Scarpa A, et al: Early epigenetic downregulation of microRNA-192 expression promotes pancreatic cancer progression. Cancer Res 76: 4149-4159, 2016.

23. Wang R, Ma Y, Yu D, Zhao J and Ma P: miR-377 functions as a tumor suppressor in human clear cell renal cell carcinoma by targeting ETS1. Biomed Pharmacother 70: 64-71, 2015.

24. Wang L, Shao J, Zhang X, Xu M and Zhao J: microRNA-377 suppresses the proliferation of human osteosarcoma MG-63 cells by targeting CDK6. Tumour Biol 36: 3911-3917, 2015.

25. Bonci D, Coppola V, Musumeci M, Addario A, Giuffrida R, Memeo L, D'Urso L, Pagliuca A, Biffoni M, Labbaye C, et al: The miR-15a-miR-16-1 cluster controls prostate cancer by targeting multiple oncogenic activities. Nat Med 14: 1271-1277, 2008. 
26. Song L, Xie X, Yu S, Peng F and Peng L: MicroRNA-126 inhibits proliferation and metastasis by targeting pik3r2 in prostate cancer. Mol Med Rep 13: 1204-1210, 2016.

27. Sun J, Fan Z, Lu S, Yang J, Hao T and Huo Q: miR-192 suppresses the tumorigenicity of prostate cancer cells by targeting and inhibiting nin one binding protein. Int J Mol Med 37: 485-492, 2016.

28. Formosa A, Markert EK, Lena AM, Italiano D, Finazzi-Agro' E, Levine AJ, Bernardini S, Garabadgiu AV, Melino G and Candi E: MicroRNAs, miR-154, miR-299-5p, miR-376a, miR-376c, miR-377, miR-381, miR-487b, miR-485-3p, miR-495 and miR-654-3p, mapped to the 14q32.31 locus, regulate proliferation, apoptosis, migration and invasion in metastatic prostate cancer cells. Oncogene 33: 5173-5182, 2014.

29. Ferracin M, Veronese A and Negrini M: Micromarkers: miRNAs in cancer diagnosis and prognosis. Expert Rev Mol Diagn 10 297-308, 2010

30. Al-Kafaji G, Al Naieb ZT and Bakhiet M: Increased oncogenic microRNA-18a expression in the peripheral blood of patients with prostate cancer: A potential novel non-invasive biomarker. Oncol Lett 11: 1201-1206, 2016.

31. Al-Kafaji G, Al-Mahroos G, Abdulla Al-Muhtaresh H, Sabry MA, Abdul Razzak R and Salem AH: Circulating endothelium-enriched microRNA-126 as a potential biomarker for coronary artery disease in type 2 diabetes mellitus patients. Biomarkers 22: 268-278, 2017.

32. Keller A, Leidinger P, Bauer A, Elsharawy A, Hass J, Backes C, Wendschlag A, Giese N, Tjaden C, Ott K, et al: Toward the blood-borne miRNome of human diseases. Nat Methods 8 : 841-843, 2011.

33. Mitchell PS, Parkin RK, Kroh EM, Frit BR, Wyman SK, Pogosova-Agadjanyan EL, Peterson A, Noteboom J, O'Briant KC Allen A, et al: Circulating microRNAs as stable blood-based markers for cancer detection. Proc Natl Acad Sci USA 105 10513-10518, 2008.

34. Weber JA, Baxter DH, Zhang S, Huang KH, Lee MJ, Galas DJ and Wang K: The microRNA spectrum in 12 body fluids. Clin Chem 56: 1733-1741, 2010.

35. Turchinovich A, Samato TR, Tonevitsky AG and Burwinkel B Circulating miRNAs: Cell-cell communication function? Front Genet 4: 119, 2013.

36. Sita-Lumsden A, Dart DA, Waxman J and Bevan CL: Circulating microRNAs as potential new biomarkers for prostate cancer. $\mathrm{Br}$ J Cancer 108: 1925-1930, 2013

37. Watahiki A, Macfarlane RJ, Gleave ME, Crea F, Wang Y, Helgason CD and Chi KN: Plasma miRNAs as biomarkers to identify patients with castration-resistant metastatic prostate cancer. Int J Mol Sci 14: 7757-7770, 2013.
38. D'Amico AV, Whittington R, Malkowicz SB, Schultz D, Blank K, Broderick GA, Tomaszewski JE, Renshaw AA, Kaplan I, Beard CJ and Wein A: Biochemical outcome after radical prostatectomy, external beam radiation therapy, or interstitial radiation therapy for clinically localized prostate cancer. JAMA 280: 969-974, 1998.

39. Al-Kafaji G, Al-Mahroos G, Alsayed NA, Hasan ZA, Nawaz S and Bakhiet M: Peripheral blood microRNA-15a as a potential biomarker for type 2 diabetes mellitus and pre-diabetes. Mol Med Rep 12: 7485-7490, 2015.

40. Schmittgen TD and Livak KJ: Analyzing real-time PCR data by the comparative C(T) method. Nat Protoc 3: 1101-1108, 2008.

41. Lu J, Getz G, Miska EA, Alvarez-Saavedra E, Lamb J, Peck D, Sweet-Cordero A, Ebert BL, Mak RH, Ferrando AA, et al: MicroRNA expression profiles classify human cancers. Nature 435: 834-838, 2005.

42. Coppola V, De Maria R and Bonci D: MicroRNAs and prostate cancer. Endocr Relat Cancer 17: F1-F17, 2010.

43. Fang YX and Gao WQ: Roles of microRNAs during prostatic tumorigenesis and tumor progression. Oncogene 33: 135-147, 2014.

44. Wang S, Aurora AB, Johnson BA, Qi X, McAnally J, Hill JA, Richardson JA, Bassel-Duby R and Olson EN: The endothelial-specific microRNA miR-126 governs vascular integrity and angiogenesis. Dev Cell 15: 261-271, 2008.

45. Fish JE, Santoro MM, Morton SU, Yu S, Yeh RF, Wythe JD, Ivey KN, Bruneau BG, Stainier DY and Srivastava D: miR-126 regulates angiogenic signaling and vascular integrity. Dev Cell 15: 272-284, 2008.

46. Miko E, Margitai Z, Czimmerer Z, Varkonyi I, Dezso B, Lányi A, Bacsó Z and Scholtz B: miR-126 inhibits proliferation of small cell lung cancer cells by targeting SLC7A5. FEBS Lett 585: 1191-1196, 2011.

47. Zhang J, Du YY, Lin YF, Chen YT, Yang L, Wang HJ and Ma D: The cell growth suppressor, mir-126, targets IRS-1. Biochem Biophys Res Commun 377: 136-140, 2008.

48. Yang X, Wu H and Ling T: Suppressive effect of microRNA-126 on oral squamous cell carcinoma in vitro. Mol Med Rep 10: 125-130, 2014.

49. Li XM, Wang AM, Zhang J and Yi H: Down-regulation of miR-126 expression in colorectal cancer and its clinical significance. Med Oncol 28: 1054-1057, 2011.

50. Sasahira T, Kurihara M, Bhawal UK, Ueda N, Shimomoto T, Yamamoto K, Kirita T and Kuniyasu H: Downregulation of miR-126 induces angiogenesis and lymphangiogenesis by activation of VEGF-A in oral cancer. Br J Cancer 107: 700-706, 2012.

51. Meister J and Schmidt MH: miR-126 and miR-126*: New players in cancer. ScientificWorldJournal 10: 2090-2100, 2010. 